\title{
NOTE ON THE THEORY OF THREE SIMILAR FIGURES.
}

BY PROFESSOR F. MORLEY.

THe theory in question is given in Casey's Sequel to Euclid (sixth edition), and also, with extensions, but still with the same geometric treatment, in the same author's Analytical Geometry (second edition). There is something to be said in favor of an appropriate analytic handling of such a theory, and I wish to give the preliminary equations in a convenient shape for such handling, the more as these equations afford a fact or two which have an air of novelty.

All letters denote complex numbers attached to points of a plane. A linear relation such as

$$
p x+q y+r=0
$$

implies that the variables $x$ and $y$ describe figures directly similar.

Two linear relations between three variables, say

$$
\left.\begin{array}{l}
p x+q y+r z+s=0, \\
p^{\prime} x+q^{\prime} y+r^{\prime} z+s^{\prime}=0,
\end{array}\right\}
$$

imply that the variables $x, y, z$ all describe similar figures.

Let

$$
\begin{array}{lll}
x=a_{1} & \text { when } & y=z=a, \\
y=b_{1} & \text { when } & z=x=b, \\
z=c_{1} & \text { when } & x=y=c .
\end{array}
$$

The double points $a, b, c$ and the invariable points $a_{1}, b_{1}, c_{1}$ suggest themselves as proper to replace the coefficients in (1). But they are too many, inasmuch as in (1) there are only four effective constants.

First introducing the double points, it is clear that the relation between $y$ and $z$ is of the form

$$
y-a=l(z-a),
$$

for it is linear, and when $y=a, z=a$.

So, also, we must have

$$
\begin{aligned}
& z-b=m(x-b) \\
& x-c=n(y-c),
\end{aligned}
$$

where $l, m, n$ are such constants that one of the three equations is a consequence of the others.

We have $y=b_{1}$ when $z=b$, so that

$$
b_{1}-a=l(b-a) \text {; }
$$


and similarly, since $z=c_{1}$ when $y=c$,

$$
c-a=l\left(c_{1}-a\right) \text {. }
$$

Therefore $\quad\left(b_{1}-a\right)\left(c_{1}-a\right)=(b-a)(c-a)$,

so that $b_{1}, c_{1} ; b, c ; a, \infty$ are pairs in involution.

Similarly

and

$$
\begin{aligned}
& c_{1}, a_{1} ; c, a ; b, \infty, \\
& a_{1}, b ; a, b ; c, \infty,
\end{aligned}
$$

are pairs in involution.

Hence we know* that there is a fourth point $d$ such that $d$ and $a_{1}$ belong to the first involution, $d$ and $b_{1}$ to the second, $d$ and $c_{1}$ to the third.

Hence $l$, or $\frac{b_{1}-a}{b-a}$, is $\frac{c-d}{b-d}$; and similarly for $m, n$.

Thus, given the double points $a, b, c$ and the point $d$, the desired equations are

$$
\left.\begin{array}{l}
\frac{y-a}{z-a}=\frac{c-d}{b-d} \\
\frac{z-b}{x-b}=\frac{a-d}{c-d} \\
\frac{x-c}{y-c}=\frac{b-d}{a-d}
\end{array}\right\}
$$

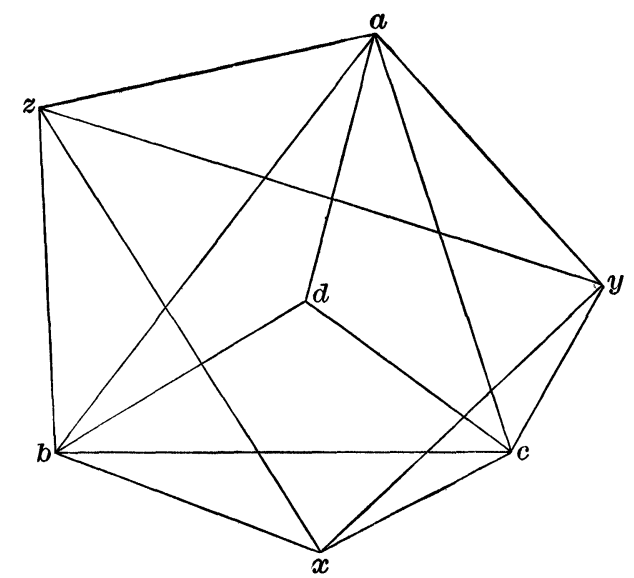

That these equations are not independent is seen at once on clearing away the denominators and adding. $\uparrow$

* Harkness and Morley, Theory of Functions, p. 32.

+ We see that $(y-a)(z-b)(x-c)=(z-a)(x-b)(y-c)$, which states that $a, x ; b, y ; c, z$ are pairs in involution; and that, taking $d$ as origin, the system (2) is solved by writing $x=b+c+b c / t$, etc., where $t$ is variable. 
The geometric statement of this is that taking any point $x$ and making

$z a y . . . . . . . b d c$,

then is $\quad y c x . . .5 . . a d b$.

Mechanical means, such as Sylvester's Plagiograph, for inducing a variable triangle to retain its shape are described in Dyck's Katalog mathematischer Modelle (Wolf, München, 1892). It is sufficient to take two such plagiographs, $x b z$, $z a y$, and connect them at $z$ while fixing the double points $b, a$ in order to have three points $x, y, z$ always describing similar figures; but the above work shows the condition that three plagiographs $x b z, z a y, y c x$, connected at $x, y, z$ and fixed at $a, b, c$, can effect the same symmetrically.

\section{NOTES.}

A Regular meeting of the American Mathematical SocIETY was held in New York, Saturday afternoon, May 25, at three o'clock, the president, Dr. Hill, in the chair. There were thirteen members present. On the recommendation of the council, Professor Clarke Benedict Williams, Kalamazoo College, Kalamazoo, Mich., was elected to membership. One nomination for membership was received. The following papers were presented:

(1) "On the limaçons which possess Poncelet polygons," by Professor F. Morley.

(2) "About biquadrate numbers whose sum is a biquadrate," by Dr. Artemas Martin.

(3) "An essay on the calculus," by Professor W. H. ECHOLS.

THE Department of Mechanics of Columbia College will give the following graduate courses during the year 1895-6: (1) Theory of the potential function, and (2) Mathematical theory of elasticity, each two hours per week throughout the year, by Professor R. S. Woodward; (3) Electromagnetic theory of light, three hours per week of first term, and (4) either Advanced thermodynamics, or Theory of sound (based on Lord Rayleigh's treatise), two hours per week throughout the year, by Professor Pupin; (5) Theoretical mechanics (based on Ziwet's Theoretical Mechanics), two hours per week throughout the year, by Mr. J. C. Pfister.

Dr. Fabian FrankLin has resigned his professorship at the Johns Hopkins University in order to assume editorial duties on the Baltimore News. This is a heary loss to the cause of pure mathematics; but we cherish the hope that the 